\title{
Single-element based ultra-wideband antenna array concepts for wireless high-precision 2-D local positioning
}

\author{
M. Gardill, G. Fischer, R. Weigel, and A. Koelpin \\ Friedrich-Alexander University Erlangen-Nuremberg, Institute of Electronics Engineering, Cauerstr. 9, \\ 91054 Erlangen, Germany
}

Correspondence to: M. Gardill (gardill@1te.eei.uni-erlangen.de)

\begin{abstract}
We generally categorize the approaches for ultrawideband antenna array design, and consequently propose simplified concepts for antenna arrays for a high-precision, ultra-wideband FMCW radar 2-D local positioning system to obtain robustness against multi path interference, perform angle of arrival analysis, as well as instantaneous heading estimation. We focus on low-cost and mechanical robust, industrial-application ready antennas. The antenna arrays are optimized for operation in the $5 \mathrm{GHz}$ to $8 \mathrm{GHz}$ frequency range and are designed towards supporting full omnidirectional $360^{\circ}$ as well as partial half-plane direction of arrival estimation. Two different concepts for vehicle- as well as wall-mounted antenna array systems are proposed and discussed. We propose a wideband unidirectional bow-tie antenna array element having $97 \%$ impedance and $37 \%$ pattern bandwidth and a robust vehicle mounted omnidirectional antenna element having more than $85 \%$ impedance and pattern bandwidth.
\end{abstract}

\section{Introduction}

With the allocation of the ultra-wideband (UWB) frequency range for unlicensed use by the FCC in 2002 interest in wideband wireless systems has increased tremendously. In particular wireless positioning systems based on round trip time of flight (RTOF) benefit from the large available continuous bandwidth, since utilizing ultra-wideband signals their reliability and accuracy can be enhanced greatly (Mahfouz et al., 2009). Whereas most commercially available UWB localization systems are based on short pulses, we focus on a novel frequency-modulated continuous waveform (FMCW) concept similar to (Roehr et al., 2008), which is extended to ultra-wideband FMCW operation in the range from $5 \mathrm{GHz}$ to $8 \mathrm{GHz}$ and high-precision direction of arrival (DOA) esti- mation techniques using multiple coherent receive channels. This allows for high-precision and robust 2-D local positioning of vehicles in various industrial scenarios by simultaneously incorporating RTOF and angle of arrival (AOA) estimations. In addition the use of a vehicular-based antenna array does allow for an instantaneous heading estimation, which cannot be achieved by single-antenna systems at all.

Besides the challenges of analog integrated circuit design, such as highly-linear and broadband FMCW ramp synthesizers and multiple coherent receive channels, the overall system performance is strongly dependent on the antennas. It is not enough to simply use antennas which offer a wide impedance bandwidth. Rather great care has to be taken of radiation characteristics such as a frequency stable pattern and a frequency- and angle-stable phase center. Subsequently, when placing the antennas in an array, the antenna elements cannot be treated as individual sensors. Mutual coupling influences the feed point impedance and the array response vector, effects such as array and substrate-guided surface waves may emerge, and a careful and angle-dependent characterization of the array is necessary. And remembering that all those challenging effects have to be treated in a wideband frequency range really does not simplify the work to be done. In addition, from McLean's limit on the $Q$ of small antennas, the inherent problem of the antenna diameter being more or less dictated by $\lambda_{\text {low }}$ (Schantz, 2003), the lowest frequency of operation, but the desired distance between two antennas in an array being optimally about $\lambda_{\text {high }} / 2$, where $\lambda_{\text {high }}$ is the highest frequency of operation, best shows the slightly paradoxical design challenge in wideband antenna arrays. In addition to those electromagnetic (EM) challenges, for the addressed industrial applications the mechanical construction of the antennas also is of major concern. They have to be robust against vibration, moisture, dust and other 


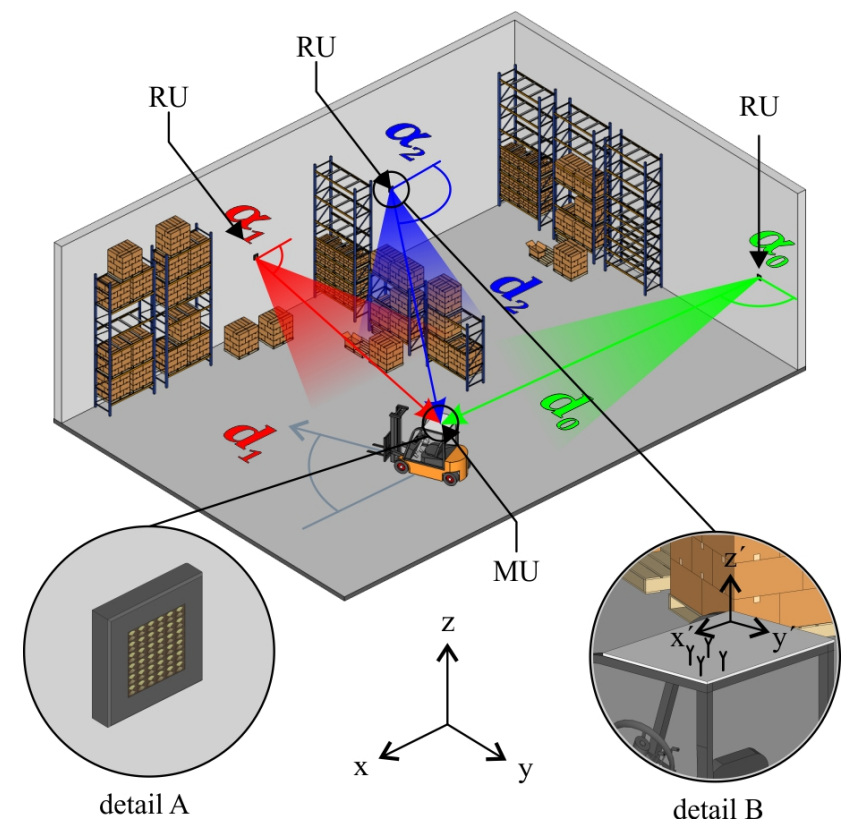

Fig. 1. Localization scenario. The position of a vehicle, e.g. fork lifter, is precisely estimated by using RTOF measurements. The novel concept of using antenna-array based systems also allows for AOA and instantaneous heading estimation. detail A: planar, wall- mounted antenna array; detail B: omnidirectional, vehicular mounted antenna array.

mechanical influences. And, of course, a low cost realization is desired.

It is obvious that antenna design for the addressed highprecision positioning system is a challenging task. This paper discusses our advances and concepts towards UWB antenna arrays for high-precision local positioning, shows some of our current development prototypes, and gives an overview on future designs. The paper is organized as follows: in Sect. 2, a detailed overview on the localization scenario and the system concept is given. In chapter 3 we then discuss our work on ultra-wideband antenna arrays. Finally in chapter 4 we draw conclusions from the preceding discussions and give an outlook on our future work.

\section{Localization scenario, system architecture, and antenna functional specification}

\subsection{Localization scenario}

Whereas a wide range of possible applications for a highprecision wireless positioning system delivering simultaneously range, angle, and heading information can be thought of, we focus, without loss of generality, on the exemplary scenario of localizing a fork lifter in a typical industrial warehouse scenario as illustrated in Figure 1. We refer to the vehicle whose position is to be estimated as the measurement unit (MU) and the units supporting the measurement process as the reference units (RU). This exemplary scenario can be applied to arbitrary positioning scenarios which obey the following restrictions in movement between MU and RU: first, there is only a translational movement between MU and RU in the $\mathrm{x} / \mathrm{y}$-plane, i.e. the $\mathrm{z}$-coordinate is fixed. Second, the rotational movements of the MU are restricted to rotations in the $\mathrm{x} / \mathrm{y}$-plane, which in particular means the $\mathrm{z}$ and $\mathrm{z}$ ' axes of the global and MU-local coordinate systems being parallel all the time.

Several wireless local positioning system configurations have been proposed over the last years (Gulden et al., 2009). We consider a typical RTOF scenario: Several RUs are mounted on precisely known positions in the localization area. In the example of Figure 1, these reference units are based on planar arrays mounted on the walls of an industrial warehouse, as shown in detail A. The object or vehicle to be positioned contains a MU, which is based on an array configuration capable of omnidirectional DOA estimation, as illustrated in detail B. Using a secondary FMCW radar concept described in Roehr et al. (2008) or more detailed in Roehr (2009), the RTOF between the MU and several RUs can be measured. A sophisticated wireless synchronization method ensures the necessary time-synchronous operation of RUs and MU (Roehr et al., 2008; Roehr, 2009). Based on the known positions of the RUs and the measured distances between MU and several RUs, a tri- or multilateration finally yields the position of the vehicle.

The use of antenna arrays and multiple coherent receive paths in both the RU and the MU offers several novel opportunities. First of all, a single antenna system is indeed capable of estimating the location of the MU, but the vehicle heading generally can only be estimated by tracking methods or using additional sensors. The use of an array at the MU allows for an instantaneous heading estimation by comparing the AOA of the signals impinging from the RU with the measured MU position and the RU positions. On the RU side, the multichannel concept may be used to include DOA estimation as an alternative to or support for MU location measurement, as a method to improve mutual RU synchronization in multipath environments, and as a possibility to detect and discard erroneous distance measurements by non-line of sight (NLOS) detection. Note that we only consider the use of multiple coherent receive $(\mathrm{RX})$ channels and the information obtained from digitally processing the RX channel outputs on both the RU and the MU side. No antenna arrays are used in the transmit (TX) direction.

\subsection{System architecture}

The system architecture of RU and MU is identical. It is illustrated in Figure 2. The actual role in the system is merely determined by the operating mode: is the device used as reference for positioning another device then it is a RU. Is it the device to be positioned then it is an MU. 


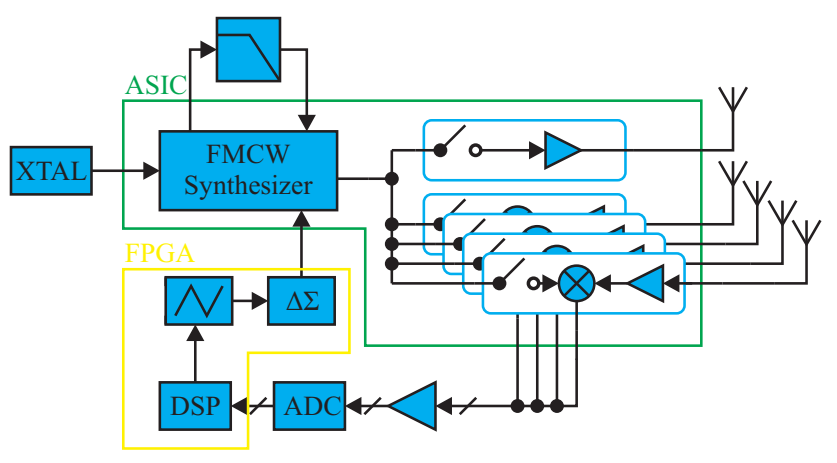

Fig. 2. System architecture of both, RU and MU. Each unit includes four RX and one TX channel. The RX channel outputs (beat signal) are sampled and handed over to an FPGA implementing the distance and DOA estimation algorithms.

The distance as well as DOA estimation is based on a wideband FMCW radar principle. The FMCW synthesizer of the RU generates a highly-linear frequency ramp in the range from $5 \mathrm{GHz}$ to $8 \mathrm{GHz}$. This ramp is transmitted using a single TX channel to the MU. The MU, which has been synchronized to the RU as described in Roehr et al. (2008) and Roehr (2009), receives the signal from the RU using the four available RX channels, mixes it down with the locally generated FMCW ramp, and outputs the amplified and low pass filtered mixing product (the beat signal) to an analog to digital converter. Frequency estimation algorithms implemented in an FPGA are used to estimate the time of flight between both stations based on the beat frequency. Mutual information between the four coherent receive channels, e.g. the phase difference, is used to perform spatial signal processing such as DOA estimation.

\subsection{Antenna functional specification}

\subsubsection{Pattern}

The pattern of the antenna array is specified in terms of individual sensor pattern, since the basic assumption of the signal processing algorithms is that the output signals of the four RX channels are obtained by sampling the impinging wave front at the position of the RX antennas, using uncoupled individual sensors. This means the pattern of each element for receiving an impinging wave front is of interest.

The desired receiving element patterns are illustrated in Figure 3. The elevation plane patterns for both the vehicle and wall-mounted antenna arrays should possess a narrow beam width to suppress parasitic multipath components due to ceiling and floor reflections. The wall mounted antenna array sensors need a unidirectional but wide-angle pattern avoiding interactions of the array with the mounting structures, e.g. a wall or the supports of a high-level rack, while enabling wide-angle RU operation and wide-angle DOA estimation. In contrast, each vehicle-mounted array sensor a)

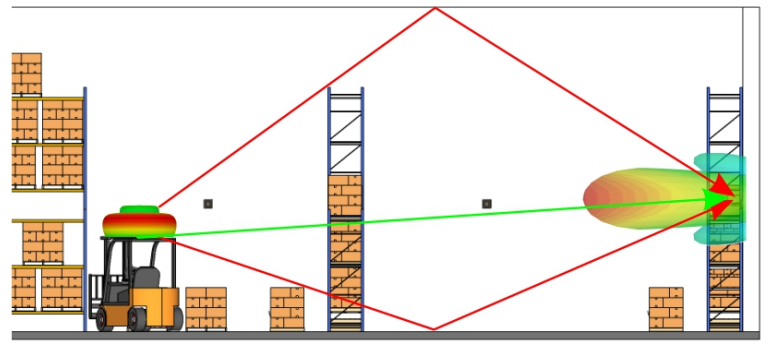

b)

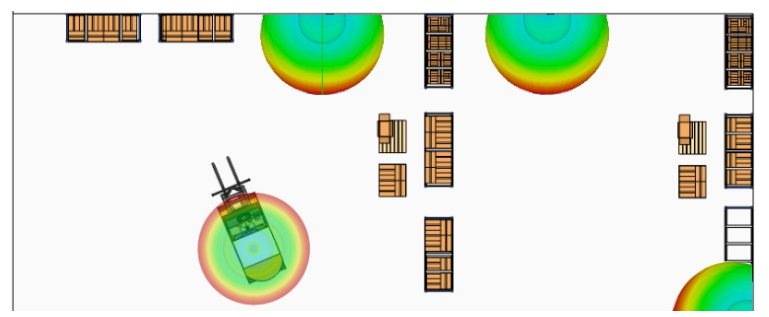

Fig. 3. Desired receiving element patterns for vehicle- and wallmounted antenna arrays. (a) view from side showing elevation pattern, (b) view from top showing azimuth pattern.

should possess a full omnidirectional azimuth radiation pattern to enable $360^{\circ} \mathrm{DOA}$ estimation and ensure proper MU operation regardless of the vehicle's heading.

\subsubsection{Polarization}

Since the $\mathrm{z}$ and $\mathrm{z}$ ' axes of the global and the MU-local coordinate systems are parallel (see Sect. 2.1) a linearly polarized system may be used. Whereas the planar wall mounted structure offers a great flexibility in polarization selection, the fact that the vehicular array will be mounted on the metallic roof of, e.g. the fork lifter, implies a benefit for vertically polarized antennas.

\subsubsection{Phase center and dispersion}

The phase center as well as the dispersive properties of an antenna are important parameters in time-reference radio positioning systems (Best, 2004) and in the particular case of FMCW systems, frequency dependent phase center movement has been reported to create a parasitic Doppler effect (Bares et al., 2003). Phase center displacement effects are closely related to dispersive effects, which are extensively treated by the impulse-based UWB community (Schantz, 2003). However, it is generally accepted that those effects are most pronounced for antennas which rely on frequencyindependent design criteria such as spiral and log-periodic antennas, which are indeed optimized to operate over a broad frequency range, but only for signals having a relatively narrow instantaneous bandwidth. Dispersive effects are generally less distinct for impulse-radiating UWB antennas such as conical or wideband dipole or monopole structures. 
Our concept of a wideband FMCW secondary radar system is somewhere in between an impulse-radiating system and a system of only narrow instantaneous bandwidth, since the FMCW sweep rate determines the transition from narrow to large instantaneous bandwidth. For practically relevant sweep rates the constraints on the time-domain properties of the antennas will never be as strict as in impulse-based systems. We thus reduce our attention to antenna elements being reported in the UWB community to have well-behaved dispersive properties, assume that the parasitic dispersive effects can be neglected in our system, and desist from an extensive treatment of dispersive properties in a first approximation. Of course, once working concepts for the antenna arrays for wideband FMCW DOA estimation have been found, a complete characterization and evaluation including phase center as well as dispersive effects is necessary.

\subsection{Ultra-wideband antenna array concepts}

Research on UWB antenna arrays can be categorized into two tiers: the classical approach which starts by selecting and optimizing an isolated wideband antenna element, then integrating the isolated element into an array, and finally accounting for mutual coupling and other parasitic effects by re-designing the antenna structure or by using compensation and calibration algorithms in the digital processing domain (Wang and Hui, 2011; Dandekar et al., 2002; Adve and Sarkar, 2000). The major problems in this approach are the fact that the dimensions of a isolated wideband antenna element are usually in the order of $\lambda_{\text {low }} / 2$, but the desired element distance $d$ for grating lobe free operation in the whole operating frequency range is $d<\lambda_{\text {high }} / 2$. In addition most of the proposed UWB antenna elements are, due to their geometrical construction, not suitable for array integration.

In the last few years a new and promising approach to UWB antenna array design has emerged, which directly utilizes the coupling between electrically small elements in an infinite array to realize ultra-wideband performance and then accounts for the truncation of the infinite array in a second step (Jones and Rawnick, 2007). Arrays based on the tightly coupled approaches have been reported to yield extremely wide impedance bandwidths, have a stable radiation pattern, due to the small element size ensure a grating-lobe free operating range up to a very high frequency, are suitable for planar, low-profile implementation, and may also be converted to conformal structures (Munk, 2003; Holland, 2011; Lee, 2007). However, since tightly coupled antenna arrays are theoretically based on an infinite periodic structure, their finite implementations also rely on a large number of elements (Holland, 2011; Holland and Vouvakis, 2010, 2012) and thus a large amount of transmit/receive (TR) modules or independent receive channels is necessary. This dramatically increases the cost and is not yet suited for civil commercial low-cost localization systems. As mentioned in Sect. 2.2, in our particular case the RF frontend is limited to only four independent receive channels. This is a wide discrepancy between the large number of required elements for a tightly coupled realization and a great challenge when considering the use of tightly-coupled arrays in the proposed localization system.

It is obvious that neither the classical isolated element based design method, nor the novel theory of UWB arrays using tightly-coupled electrically small elements provides an out-of-the-box solution. We generally focus on the examination and study of both concepts, but restrict the focus onto simplified isolated-element based UWB antenna arrays in this contribution.

The antenna arrays discussed in this section are based on wideband antenna elements, which in a first step are designed and optimized in isolation, and then incorporated into an antenna array. After a preliminary study of the vast amount of UWB antenna elements available in literature, based on the antenna functional specification from Sect. 2.3 two most promising antenna elements have been selected as possible candidates. These are the famous Bow Tie antenna element for a planar, wall-mounted array, and the monocone antenna element for the omnidirectional vehicle array. Those basic concepts have been optimized for the target application needs and novel design modifications are discussed in the following section.

\subsubsection{The planar wideband reflector backed bow tie with pattern stabilization}

A proposed antenna element for an isolated-element based wall-mounted antenna array is a Bow Tie antenna printed on a dielectric substrate, above a planar reflector. A mechanical robust and low-cost separation of antenna and reflector is achieved by using a readily available PTFE block of thickness $t$. The planar reflector is in turn printed on a second dielectric substrate and simultaneously acts as ground plane for the feed network printed on the opposing side of the reflector substrate. This layer stack-up is illustrated in Figure 4. The antenna is fed from the back side by using an impedance controlled two-wire transmission line, realized by two silver plated copper wires press fitted into precisely drilled holes and penetrating the ground plane reflector through an rectangular aperture. On both the antenna and the feed network layer the feed lines are soldered to the antenna and feed network metallization, respectively. Whereas an unlimited variety of antenna metallization shapes is available and their benefits and drawbacks have been extensively studied in literature, we focus on a rounded-edge Bow Tie antenna (REBA), since this has been reported to yield good return loss results (Qu and Ruan, 2006) and simplifies the design by reducing the antenna metallization degrees of freedom to merely three: the opening angle $\alpha$, the Bow Tie radius $r$, and the gap width $w$. The entire antenna element has several additional degrees of freedom which are the PTFE thickness $t$, the twin wire feed line diameter $d$ and conductor separation $s$, and the 

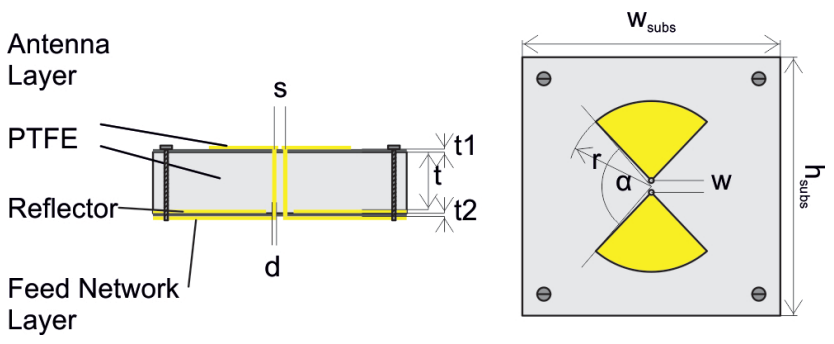

Fig. 4. Geometry and layer stack up of proposed Bow Tie antenna element. The groundplane of the feed network layer simultaneously acts as antenna reflector. a)

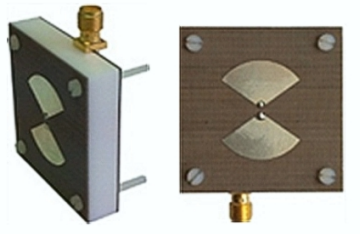

c)

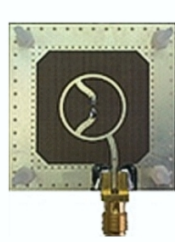

d)

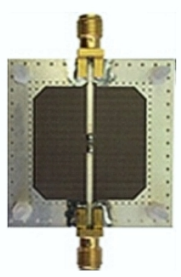

Fig. 5. Fabricated Bow Tie antenna element. (a) Perspective view, (b) view from top, (c) feed network metallization including balun and d) antenna using differential feed.

substrate thicknesses $t_{1}$ and $t_{2}$ of antenna and feed network substrates, respectively.

The Bow Tie antenna in free space, as well as on a dielectric half space, has been treated extensively in literature. In this conceptual-focused paper we omit a detailed discussion and refer the reader to Compton et al. (1987) and Allen et al. (2007). To couple out the radiated power into the air we use a planar reflector below the substrate. This is the classical approach to convert a bidirectional antenna into a unidirectional one. However, in contrast to narrowband antennas where typically an antenna-reflector spacing of $t=\lambda_{\mathrm{d}} / 4$ is used, there is really no optimum spacing for wideband antennas above ground: In the low frequency limit, i.e. for a small antenna reflector spacing, the current in the antenna is shorted due to the reflector currents, the radiation resistance is lowered and hence the input impedance is strongly influenced. In the high frequency limit, i.e. for a large antenna reflector spacing, the input impedance is less altered, but for a spacing larger than $\lambda_{\mathrm{d}} / 4$ the pattern in broadside direction breaks down until having a deep null at an antenna reflector spacing of $\lambda_{\mathrm{d}} / 2$ , compare the far field simulation results from Figure 7 and note the breakdown in broadside direction of about $6 \mathrm{~dB}$ for $8 \mathrm{GHz}$.

Our solution to this inherent problem is as follows: whereas the simplified theory of reflector backed antennas is based on infinitely large reflectors, each physical realization will, of course, use a reflector of finite extend. On the edges of the finite-size reflector diffractions will occur, which
Table 1. Optimized parameters of Bow Tie antenna element. All dimensions are given in $\mathrm{mm}$, angles in degree.

\begin{tabular}{cccccccc}
\hline$r$ & $\alpha$ & $w$ & $h_{\text {subs }}$ & $w_{\text {subs }}$ & $t$ & $s$ & $d$ \\
\hline 12 & 68 & 1.6 & 40 & 40 & 8.5 & 1.8 & 0.8 \\
\hline
\end{tabular}

usually have a parasitic effect on the antenna pattern and often are tackled by using absorbing materials at the reflector edges. The diffracted field can, in a first approximation, be thought of as being generated from line currents flowing on the edges of the reflector. When limiting the size of the reflector to a width corresponding to about $w_{\text {subs }}=\lambda_{\text {high }}$, the wavelength at the highest frequency of operation, the fields radiated from the in-phase currents on the left and right edges will counteract the emerging breakdown in broadside direction and stabilize the radiation pattern, while leaving the impedance characteristics of the antenna nearly untouched. Hence the antenna can be placed at a larger distance than $\lambda_{\mathrm{d}, \mathrm{high}} / 4$ from the reflector, without occurrence of the pattern breakdown in broadside direction. However, the radiation from the truncated reflector edges also has a negative effect: the front to back ratio of the pattern is severely degraded. Nevertheless this approach clearly shows that introducing two in-phase line currents, neighbored at a distance of about $\lambda_{\text {high }} / 2$ to the Bow Tie center, the pattern can be stabilized. Using the above mentioned theory the antenna element was optimized for the frequency range from $5 \mathrm{GHz}$ to $8 \mathrm{GHz}$ and several prototypes were manufactured. The optimized antenna dimensions are given in Table 1.

Figure 6 shows the simulated and measured return loss of the optimized antenna element when using a differential feed structure as well as a simple rat-race based balun, as shown in Fig. 5, respectively. Using the differential feed a $-10 \mathrm{~dB}$ return loss range from $5 \mathrm{GHz}$ to $14.5 \mathrm{GHz}$, i.e. a $97 \%$ impedance bandwidth, is achieved. The rat-race balun limits the usable bandwidth to the range of $5 \mathrm{GHz}$ to $8 \mathrm{GHz}$, but still meets the requirements of our target application. An excellent agreement between simulated and measured results is obvious. Radiation pattern simulations and measurements for broadside direction are shown in Fig. 7. The stability of the pattern in broadside direction over frequency, as well as the successful avoidance of the pattern breakdown in the highfrequency limit is obvious.

A four-element linear array has been built from the planar wideband reflector backed Bow Tie antennas by mounting the antenna elements on a laser-cut acrylic plate. An element distance of $d=46.567 \mathrm{~mm}$ corresponding to a $0.9 \lambda$ distance at $f=5.794 \mathrm{GHz}$ was selected. The distance selection was based on two facts: firstly, the novel FMWC DOA estimation algorithm uses the start frequency of the FMCW ramp as reference for the DOA estimation. Since the initial test system is operating in the $5.8 \mathrm{GHz}$ ISM band, the above mentioned 


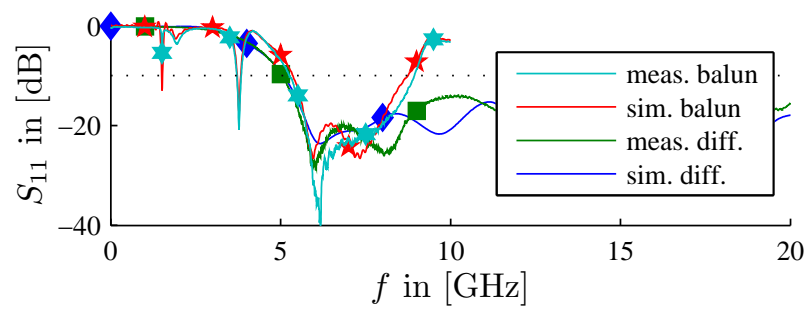

Fig. 6. Measured return loss of proposed Bow Tie antenna. $-10 \mathrm{~dB}$ return loss range using differential feed is from $5 \mathrm{GHz}$ to $14.5 \mathrm{GHz}$, i.e. $97 \%$ bandwidth. Rat-race limits useable bandwidth from $5 \mathrm{GHz}$ to $8 \mathrm{GHz}$.
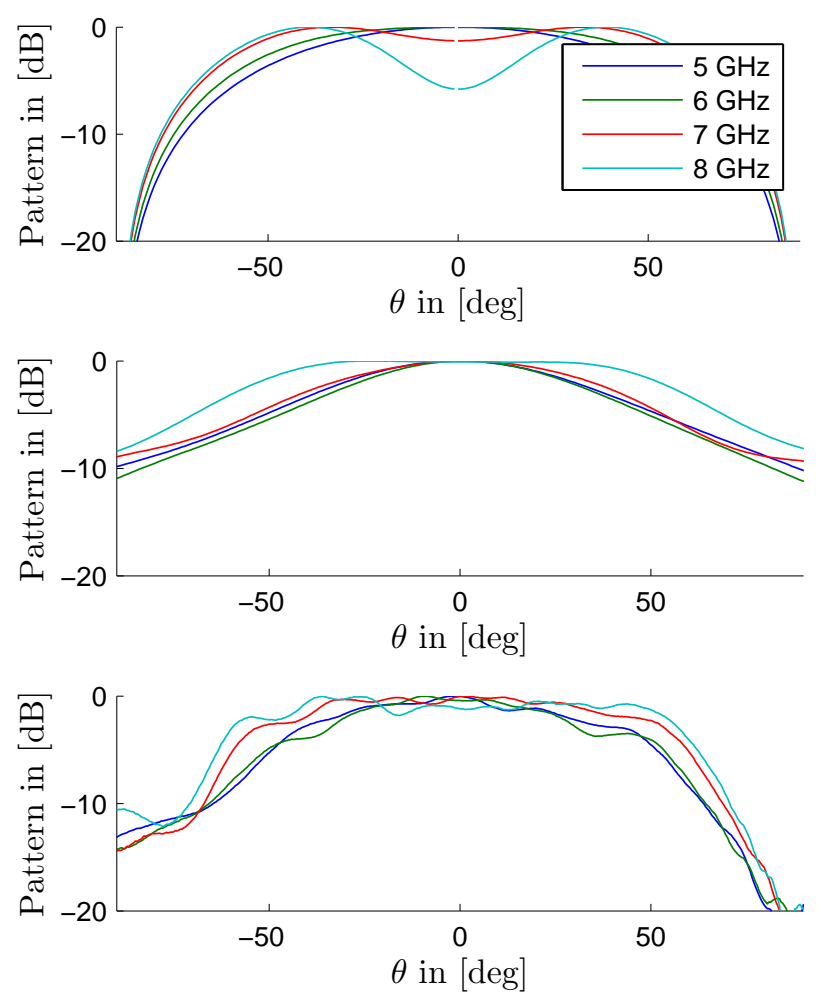

Fig. 7. Simulated and measured H-plane patterns of proposed Bow Tie antenna. (top) Simulation on infinite ground plane showing pattern breakdown at high-frequency limit, (center) simulation results on truncated reflector showing breakdown compensation, and (bottom) measurements of proposed element proving breakdown compensation.

reference frequency results. Secondly, the desired range of ambiguity-free DOA estimation has been lowered to a range of $45^{\circ}$ from broadside direction. This allows to use the relatively large elements with an element spacing up to $\lambda$. A photograph of the prototype array is included as inset in Fig. 8 and the simulated 4-port S-parameter results are given in the plot of Fig. 8. The simulation results show a good impedance match in the desired operating frequency range from $5 \mathrm{GHz}$

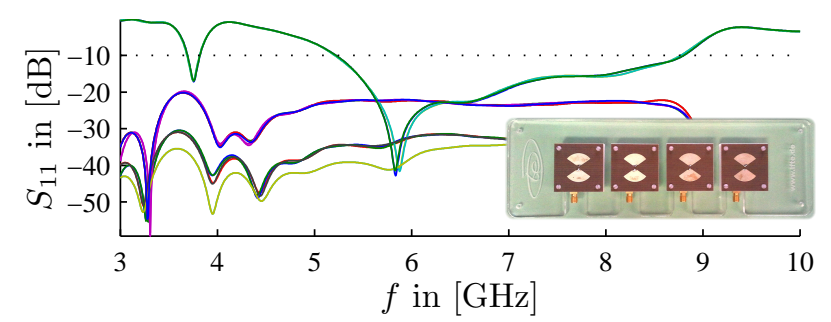

Fig. 8. Simulated four-port S-Parameters of Bow Tie antenna array using differential feed. Return loss is marginally altered, transmit mode coupling is below $-20 \mathrm{~dB}$.

to $8 \mathrm{GHz}$ and an acceptable transmit mode coupling of maximum $-20 \mathrm{~dB}$ to neighbored elements. A full measurement characterization of the prototype array in receive mode is ongoing.

In conclusion, the Bow Tie based antenna array concept may be suitable if the desired ambiguity-free DOA estimation range can be limited to $\pm 45^{\circ}$ from broadside direction, since the width $w_{\text {subs }}$ of the wideband elements is in the order of a wavelength at the lowest frequency of operation. While not inherently offering a narrow elevation pattern, this can be achieved by connecting several vertically stacked Bow Tie elements in phase. Still some work has to be done on integrating the antenna elements onto a common substrate and above a common large reflector. In particular this means that the pattern stabilization mechanism, which is based on the reflector truncation, has either to be omitted, or implemented by means of another stabilization method.

\subsubsection{The monocone above ground}

The theory of Bow Tie antennas and monocone antennas essentially has the same origin and their electromagnetic behavior is comparable to a great extent. Monocone structures above ground planes can be derived from biconical structures using image theory, which have been extensively treated by Schelkunoff (1951). Planar Bow Tie structures in turn can be analytically treated by deriving their characteristic modes in a sphero-conal coordinate system degraded to its planar equivalent (Stockbroeckx and Vander Vorst, 2000). Hence both antenna types are based on the same radiation mechanism, which idealizes them for a paired used in the proposed FMCW localization system. Again we skip a detailed treatment of monocone structures in this paper, refer the interested reader to the provided literature, and discuss the conceptual benefits and the optimized antenna element in this section.

Although monoconical structures are one of the oldest antenna elements - the first one was already used by Marconi at the radio station in Poldhu, Cornwall (Simons, 1996) they still are subject to current research and due to their wide bandwidth became a quasi-reference in UWB antenna 


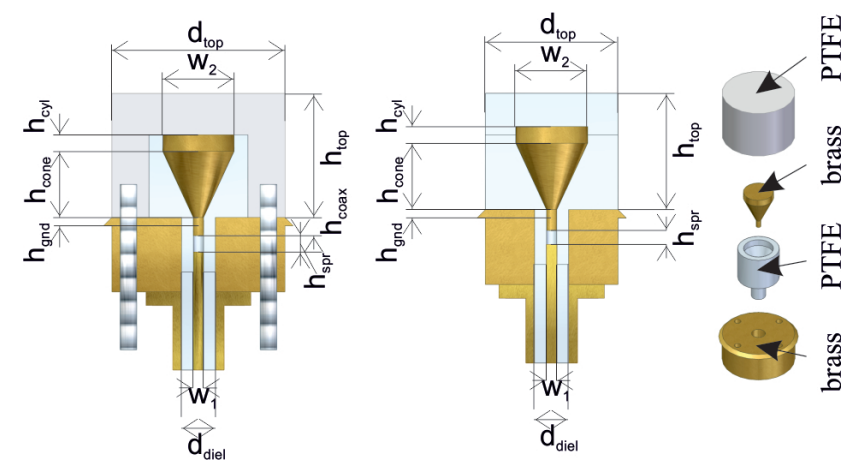

Fig. 9. Technical drawings of proposed monocone antennas Type1 (left), Type2 (center), and exploded view of Type 1 (right).

designs. Recent publications are often dealing with improving the electromagnetic characteristics (Aten and Haupt, 2009; Taniguchi and Kobayashi, 2003; Hu et al., 2011), but an important fact which often is neglected is the design of practically relevant antenna structures which cover both, desired electromagnetic radiation characteristics as well as a robust mechanical construction protecting the antenna from environmental influence such as dust, moisture, vibrations and other mechanical influences, and enabling it to be mounted on various objects. Since every additional structure introduced into the area surrounding an antenna changes its electromagnetic behavior, the aforementioned practically relevant characteristics are not simply a problem of mechanical design, but detailed and in-depth treatment of electromagnetic and mechanical characteristics is necessary.

For the development of a simplified isolated-antenna element based vehicle mounted antenna array, we concentrate our research onto the development of a practically relevant monocone antenna, which can easily be manufactured, mounted, and offers the necessary mechanical stability. A technical drawing of the proposed monocone antenna is given in Fig. 9. The basic construction of the proposed monocone antenna, referred to as Type 1 , is illustrated in Fig. 9. The key concept of the antenna is a metallic conical structure of height $h_{\text {cone }}$ which has two cylindrical extensions on the top and the bottom, with heights $h_{\text {cyl }}$ and $h_{\text {coax }}$, respectively. The upper cylindrical extension is used for impedance tuning and serves as a fixture against lateral movements in a second version of the monocone antenna, referred to as type 2 (Fig. 9). The lower cylindrical extension is inserted into a hole drilled in the antenna socket. The socket acts as the ground plane, or counterpoise system, and as a fixture for the antenna module. To fix the cone in the right position above the socket two PTFE parts are used. The inner PTFE part has an exact imprint of the cone, such that the cone can be pressed into it and is securely fixed. The lower cylindrical extension of the inner PTFE part is then tightly pressed into the hole drilled into the socket and thus precisely aligns the axes of the cone lower cylindrical extension, inner PTFE part

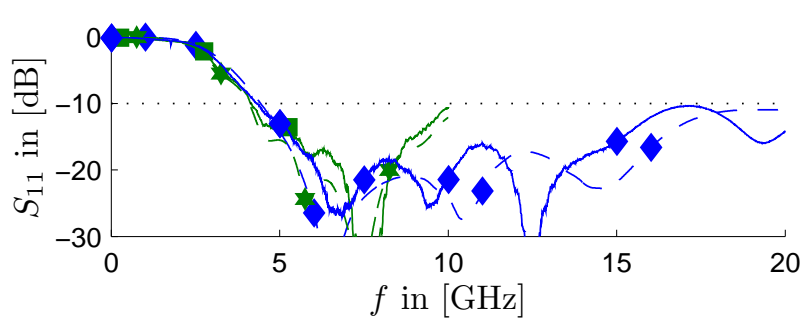

Fig. 10. Simulated (dashed lines) and measured (solid lines) return loss results for antenna Type 1 (green) and antenna Type 2 (blue).

cylindrical extension, and the hole drilled in the socket. The lower parts form a coaxial structure which is used to feed the antenna element. To protect the antenna structure from environmental influences the outer PTFE cylinder is used. A holed drilled in the cylinder exactly matches the outer diameter of the inner PTFE part and when putting over the outer PTFE part over the combination of inner PTFE and cone, a tightened unit is created. This unit is then inserted into the socket, compare the explosion drawing from Fig. 9. The construction is inherently self-fixed against lateral movements, but a vertical fixture of all parts has to be ensured. This is realized by means of nylon threaded rods, which are screwed into four threads in the outer PTFE cylinder, which penetrate the socket through four drills, and which are then locked on the bottom side of the socket with nylon nuts. This method of fixing the antenna structure increases the antenna dimensions, since minimum necessary material thicknesses for the screws, the drills, and the threads have to be taken care of. In addition the nylon screws introduce an inhomogeneity into the dielectric near the cone apex disturbing the outward propagating spherical waves and thus have a negative influence on the antenna characteristics.

Hence, the monocone Type 2 was developed. Whereas it is generally based on the same construction principles, it ensures the vertical stability of all components using adhesive connections which can be realized by pretreatment of the PTFE surfaces with a reactive process gas or an n-Heptane based liquid primer. Without the need for nylon bolts the antenna can be reduced in size and its characteristics can be optimized. In addition the adhesive serves as seal between the antenna parts and realizes an effective protection against moisture and dust.

From the bottom of the socket a flange-mount SMA connector with extended PTFE dielectric and extended inner conductor is used to interface the antenna. As can be seen from the technical drawings, the dielectric from the connector and antenna are pressed against each other, where the low flexural strength of PTFE ensures a tight connection. In contrast it can be seen that a small gap of height $h_{\text {spr }}$ between the center conductors of the coaxial construction exist. This gap is filled with golden a spring-like mesh (a FuzzButton), which is tightly pressed against the conductors 
Table 2. Optimized geometry of monocone antennas Type 1 and Type 2. All dimensions are in millimeters.

\begin{tabular}{cccccccc}
\hline$h_{\text {gnd }}$ & $h_{\text {cone }}$ & $h_{\text {cyl }}$ & $h_{\text {coax }}$ & $w_{1}$ & $w_{2}$ & $d_{\text {top }}$ & $d_{\text {diel }}$ \\
\hline 1 & 8.1 & 2 & 2.2 & 1.25 & 8.67 & 21 & 4.1 \\
1 & 8.1 & 2 & 2.2 & 1.25 & 8.67 & 16 & 4.1 \\
\hline
\end{tabular}
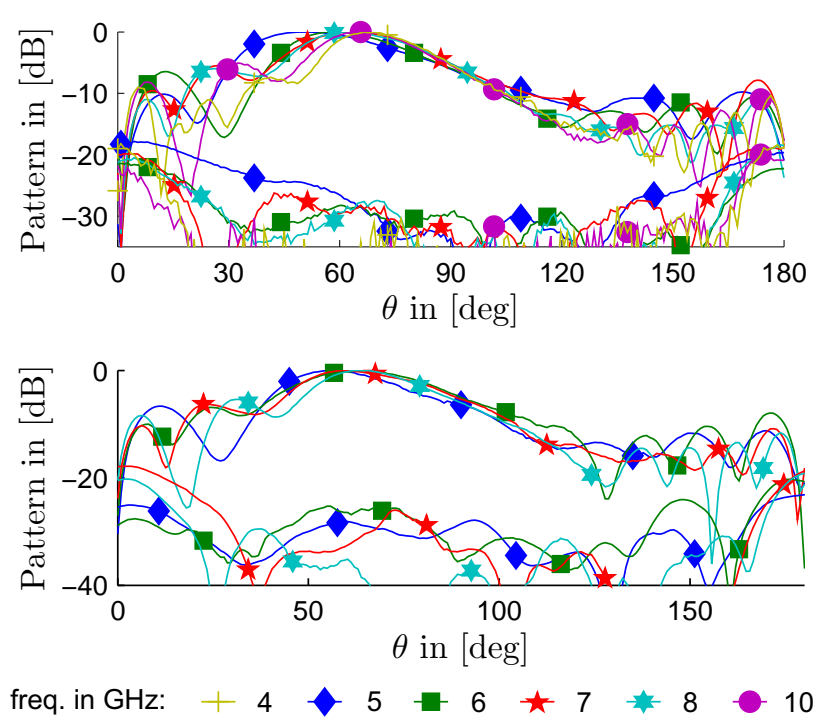

Fig. 11. Measured Co-polar and X-polar elevation pattern of Type 1 (top) and Type 2 (bottom) antennas, respectively. Groundplane size $d_{\text {plate }}=300 \mathrm{~mm}$.

of SMA connector and cone and thus ensures a reliable coaxial connection avoiding any imperfections in the feed line impedance.

Starting from rough estimates on the necessary dimensions of the conical structure obtained from theory and previous examinations on biconical structures, we numerically optimized the antenna geometry in the frequency range from $5 \mathrm{GHz}$ to $8 \mathrm{GHz}$ using a commercially available electromagnetics software package. The optimized values are given in Table 2.

Return loss simulation and measurement results for both the Type 1 and Type 2 antenna variants are given in Fig. 10 and farfield patterns in Fig. 11. Since the antenna is designed to operate above a ground plane such as the roof of a vehicle, the antenna is inserted into a metallic plate of diameter $d_{\text {plate }}$. Where according to image theory the ideal pattern should have a maximum along the ground plane, the effects of edge diffractions on the finite ground plane result in the pattern maximum at about $\theta \approx 30^{\circ}$, compare Fig. 11. As stated in Sect. 2.3.1 this is indeed not optimum, but inherently based on the ground plane size. The larger the ground plane, the smaller the elevation of the pattern maximum becomes.
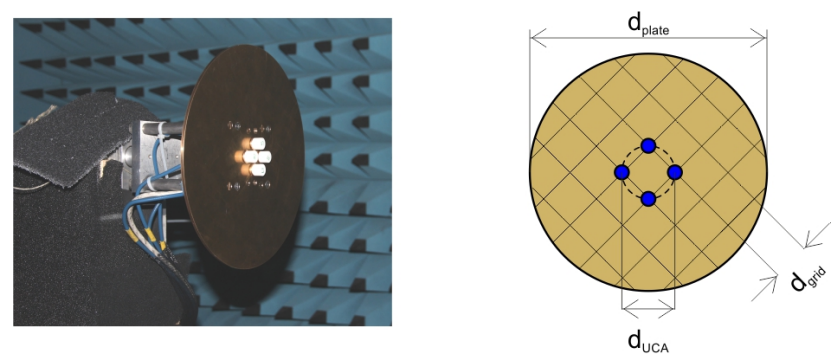

Fig. 12. Photograph and geometry of four-element monocone UCA.

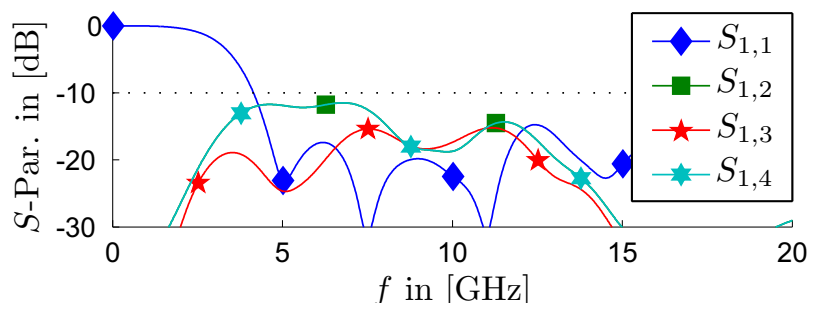

Fig. 13. Simulated four-port S-Parameters of Type 2 Monocone antenna array embedded in groundplane of diameter $d_{\text {plate }}=300 \mathrm{~mm}$. In comparison to Bow Tie antenna, larger transmit mode coupling is obvious.

The proposed monocone antenna elements can be used to build arbitrary planar array geometries above a ground plane, whereas the Type 2 element adds, due to its reduced diameter, more flexibility and allows for smaller element spacings. For example, Fig. 12 shows a photograph as well as the geometry of a four-element uniform circular array with an grid spacing of $d_{\text {grid }}=25 \mathrm{~mm}$, corresponding to $\lambda / 2$ at $6 \mathrm{GHz}$.

Figure 13 shows the simulated S-Parameter results for the linear array with $d=25 \mathrm{~mm}$. Compared to the linear Bow Tie array, larger mutual coupling is obvious.

The proposed Monocone element promises to be a suitable element for vehicle-based wideband arrays with flexible array configurations. A detailed characterization of various array structures and the parasitic effect of mutual coupling, as well as possible compensation methods will follow.

\section{Conclusions}

We discussed the use of and requirements on vehicle- and wall-mounted antenna arrays for DOA estimation in a novel high-precision FMCW Radar 2-D local positioning system. We categorized the concepts of UWB antenna array design and discussed two simplified isolated antenna element approaches in detail. Simulation results and where available measurements have illustrated the viability of both approaches.

Much research work still has to be done towards fullyintegrated antenna arrays for UWB DOA estimation for the target application. The planar Bow Tie elements need to be 
integrated on a common substrate and therefore the method for pattern stabilization has to be improved. The proposed monocone antennas are a useful and practical antenna design, but a further improvement of their elevation-plane radiation pattern is desirable.

Acknowledgements. This research project is funded by the Bavarian Ministry of Economic Affairs, Infrastructure, Transport and Technology, project. No. IUK-0908-0004 and the European Regional Development Fund (ERDF).

\section{References}

Adve, R. S. and Sarkar, T. K.: Compensation for the effects of mutual coupling on direct data domain adaptive algorithms, IEEE T. Antenn. Propag., 48, 86-94, 2000.

Allen, B., Dohler, M., Okon, E., Malik, W., Brown, A., and Edwards, D.: Ultra-wideband: Antennas and Propagation for Communications, Radar and Imaging, Wiley, Chichester, West Sussex, England, 2007.

Aten, D. and Haupt, R.: Genetically optimized, low profile, wideband, shorted monocone antenna, in: Antennas and Propagation Society International Symposium, 2009. APSURSI '09. IEEE, 1-4, doi:10.1109/APS.2009.5171992, 2009.

Bares, C., Brousseau, C., Le Coq, L., and Bourdillon, A.: Effect of antenna phase center displacement on FM-CW measurements \& application to radar system, in: Antennas and Propagation Society International Symposium, 2003, IEEE, 3, 812-815, doi:10.1109/APS.2003.1220034, 2003.

Best, S.: Distance-measurement error associated with antenna phase-center displacement in time-reference radio positioning systems, Antennas and Propagation Magazine, IEEE, 46, 13-22, doi:10.1109/MAP.2004.1305530, 2004.

Compton, R., McPhedran, R., Popovic, Z., Rebeiz, G., Tong, P., and Rutledge, D.: Bow-tie antennas on a dielectric half-space: Theory and experiment, IEEE T. Antenn. Propag., 35, 622-631, doi:10.1109/TAP.1987.1144162, 1987.

Dandekar, K. R., Ling, H., and Xu, G.: Experimental study of mutual coupling compensation in smart antenna applications, IEEE T. Wirel. Commun., 1, 480-487, doi:10.1109/TWC.2002.800546, 2002.

Gulden, P., Roehr, S., and Christmann, M.: An overview of wireless local positioning system configurations, in: Proc. IEEE MTTS Int. Microwave Workshop Wireless Sensing, Local Positioning, and RFID IMWS, 1-4, doi:10.1109/IMWS2.2009.5307894, 2009.

Holland, S. and Vouvakis, M.: A $7-21 \mathrm{GHz}$ Planar Ultrawideband Modular Array, in: Antennas and Propagation Society International Symposium (APSURSI), IEEE, 1-4, doi:10.1109/APS.2010.5561424, 2010.
Holland, S. and Vouvakis, M.: The Planar Ultrawideband Modular Antenna (PUMA) Array, IEEE T. Antenn. Propag., 60, 130-140, doi:10.1109/TAP.2011.2167916, 2012.

Holland, S. S.: Low-profile, Modular, Ultra-Wideband Phased Arrays, Paper 450, Open Access Dissertations, 2011.

$\mathrm{Hu}$, Z. H., Hall, P. S., Kelly, J. R., and Gardner, P.: Wideband omni conical monopole antenna with high $\mathrm{Q}$ band-notched behaviour, in: Proc. Int Antenna Technology (iWAT) Workshop, 37-40, 2011.

Jones, M. and Rawnick, J.: A New Approach to Broadband Array Design using Tightly Coupled Elements, in: Military Communications Conference, 2007. MILCOM, IEEE, 1 -7, doi:10.1109/MILCOM.2007.4454764, 2007.

Lee, J. J.: Ultra Wideband Arrays, in: Antenna Engineering Handbook, edited by Volakis, J. L., chap. 24, 1-24, Mcgraw-Hill Professional, New York, NY, USA, 2007.

Mahfouz, M. R., Fathy, A. E., Kuhn, M. J., and Wang, Y.: Recent trends and advances in UWB positioning, in: Proc. IEEE MTT-S Int. Microwave Workshop Wireless Sensing, Local Positioning, and RFID IMWS, 1-4, 2009.

Munk, B.: Finite Antenna Arrays and FSS, Wiley, 2003.

Qu, B. S.-W. and Ruan, C.-L.: Effect of round corners on bowtie antennas, Progress In Electromagnetics Research, 57, 179-195, 2006.

Roehr, S.: System-Theoretic Analysis and Optimization of a Novel Secondary Radar Concept for Precise Distance and Velocity Measurement, Logos Verlag Berlin, 2009.

Roehr, S., Gulden, P., and Vossiek, M.: Precise Distance and Velocity Measurement for Real Time Locating in Multipath Environments Using a Frequency-Modulated Continuous-Wave Secondary Radar Approach, IEEE T. Microw. Theor., 56, 23292339, doi:10.1109/TMTT.2008.2003137, 2008.

Schantz, H. G.: Introduction to ultra-wideband antennas, in: Proc. IEEE Conf. Ultra Wideband Systems and Technologies, 1-9, doi:10.1109/UWBST.2003.1267792, 2003.

Schelkunoff, S. A.: Electromagnetic waves, The Bell Telephone Laboratories series., Van Nostrand, Toronto (u.a.), 7. print. edn., the Bell Telephone Laboratories series, 1951.

Simons, R. W.: Guglielmo Marconi and Early Systems of Wireless Communication, General Electric Company Review, 11, 37-55, 1996.

Stockbroeckx, B. and Vander Vorst, A.: Electromagnetic modes in conical transmission lines with application to the linearly tapered slot antenna, IEEE T. Antenn. Propag., 48, 447-455, doi:10.1109/8.841906, 2000.

Taniguchi, T. and Kobayashi, T.: An omnidirectional and lowVSWR antenna for the FCC-approved UWB frequency band, in: IEEE Antenn. Propag., 3, 460-463, 2003.

Wang, B. H. and Hui, H. T.: Wideband mutual coupling compensation for receiving antenna arrays using the system identification method, IET Microw. Antenna. P., 5, 184-191, doi:10.1049/ietmap.2010.0120, 2011. 